\title{
Corrigenda
}

\section{Complexes of Aminobenzylamines. Part I. Complexes of $o$-Aminobenzylamine with Copper(II), Cobalt(II) and Nickel(II)}

\author{
Dimitra Kovala-Demertzi, John Michael Tsangaris and Nick Hadjiliadis*
}

University of Ioannina, Department of Chemistry, Inorganic Chemistry Laboratory, Domboli 31, Ioannina, Greece

\section{Experimental}

Materials and methods

Anhydrous $\mathrm{CuCl}_{2}, \mathrm{CuBr}_{2}, \mathrm{NiCl}_{2}, \mathrm{NiBr}_{2}, \mathrm{CoCl}_{2}, \mathrm{CoBr}_{2}$ together with $\mathrm{NiCl}_{2} \cdot 6 \mathrm{H}_{2} \mathrm{O}, 2$-aminobenzenonitrile and $\mathrm{Li}\left[\mathrm{AlH}_{4}\right]$ were purchased from Fluka, ROC/RK Merck and used without further purification.

I.r. spectra were recorded on Perkin-Elmer 577 and 597 spectrophotometers, in $\mathrm{KBr}$ pellets or Nujol mulls on $\mathrm{KBr}$ plates. Diffuse reflectance spectra were recorded in a Varian 634 spectrophotometer by diluting the samples with $\mathrm{MgO}$. U.v.-vis. spectra were recorded on Bosch-Lomb-Shimadji Spectronic $210 \mathrm{~W}$, Hitachi $100-70$ and Cary 17 spectrophotometers using $1 \mathrm{~cm}$ rectangular quartz cells. E.s.r. spectra were recorded on a Varian Y-4502 Spectrometer using DPPH as internal reference. Molar conductance measurements were performed on a Metrohm Ltd., Herisau E-527 conductoscope. Magnetic susceptibility measurements were made by the Fara- day method using $\mathrm{Hg}\left[\mathrm{Co}(\mathrm{CNS})_{4}\right]_{2}$ as reference. M.ps. are uncorrected. $\mathrm{C}, \mathrm{H}$ and $\mathrm{N}$ elemental analyses were performed by Dr. Mantzos of the National Hellenic Research Foundation, in Athens. Halogens were determined potentiometrically or by precipitation as $\mathrm{AgX}(\mathrm{X}=\mathrm{Cl}$ or $\mathrm{Br})$. Metal analyses was made by following the appropriate method either titrimetrically or by precipitation ${ }^{(48)}$.

\section{Preparation of the complexes}

All the complexes described above, were first dried at room temperature in a vacuum desiccator containing $\mathrm{CaCl}_{2}$. The drying was continued to constant weight at $90^{\circ} \mathrm{C}$ under vacuum over $\mathrm{P}_{2} \mathrm{O}_{5}$.

The deuterated $o$-aba and its complexes were prepared by shaking small quantities with $\mathrm{D}_{2} \mathrm{O}$ for $24 \mathrm{~h}$.

The ligand $o$-aba was prepared according to the method of Amundsen and Nelson ${ }^{(49)}$.

The preparation of the complexes is briefly described in the Table.

\begin{tabular}{|c|c|c|c|c|c|}
\hline Complex & Starting materials & Solvents. & $\begin{array}{l}\text { Reaction time } \\
\text { and temperature }\end{array}$ & $\begin{array}{l}\text { Washing the } \\
\text { precipitate }\end{array}$ & $\begin{array}{l}\text { Yield } \\
\%\end{array}$ \\
\hline $\mathrm{CuLCl}_{2}$ & $\begin{array}{l}\mathrm{CuCl}_{2} \text { (anhydrous) (4 mmols) } \\
+\mathrm{Ligand}(2 \mathrm{mmols})\end{array}$ & $\begin{array}{l}\text { EtOH }+ \text { EtOAc, } 1: 1 \\
\text { proportion }\left(20 \mathrm{~cm}^{3}\right)\end{array}$ & $\begin{array}{c}5 \mathrm{~h} \\
\left(25^{\circ} \mathrm{C}\right)\end{array}$ & $\begin{array}{l}\text { EtOAc + petroleum } \\
\text { ether }\end{array}$ & 90 \\
\hline $\mathrm{CuL}_{2} \mathrm{Cl}_{2}$ & $\begin{array}{l}\mathrm{CuCl}_{2} \text { (anhydrous) (2 mmols) } \\
+\mathrm{L}(4.4 \text { mmols })\end{array}$ & $\begin{array}{l}\mathrm{EtOH}+\mathrm{EtOAc}, 1: 2 \\
\text { proportion }\left(30 \mathrm{~cm}^{3}\right)\end{array}$ & $\begin{array}{l}5 \mathrm{~h} \\
\left(25^{\circ} \mathrm{C}\right)\end{array}$ & $\begin{array}{l}\text { EtOAc + petroleum } \\
\text { ether }\end{array}$ & 90 \\
\hline $\mathrm{CuL}_{2} \mathrm{Br}_{2}$ & $\begin{array}{l}\mathrm{CuCl}_{2} \text { (anhydrous) }(2 \mathrm{mmols}) \\
+\mathrm{L}(4.4 \text { mmols })\end{array}$ & $\begin{array}{l}\mathrm{MeOH} \cdot \mathrm{EtOAc}, 1: 2 \\
\text { proportion }\end{array}$ & $\begin{array}{l}24 \mathrm{~h} \\
\left(25^{\circ} \mathrm{C}\right)\end{array}$ & $\begin{array}{l}\mathrm{MeOH}+\text { petroleum } \\
\text { ether }\end{array}$ & 90 \\
\hline $\mathrm{CoLX}_{2}$ & $\begin{array}{l}\mathrm{CoX}_{2} \text { (anhydrous) (4-6 mmols) } \\
+\mathrm{L}(2 \mathrm{mmols})\end{array}$ & $\mathrm{EtOH}+\mathrm{EtOAc}\left(20 \mathrm{~cm}^{3}\right)$ & $\begin{array}{l}24 \mathrm{~h} \\
\left(25^{\circ} \mathrm{C}\right)\end{array}$ & $\begin{array}{l}\text { EtOAc + petroleum } \\
\text { ether }\end{array}$ & 70 \\
\hline $\mathrm{CoL}_{2} \mathrm{X}_{2}$ & $\begin{array}{l}\mathrm{CoX}_{2} \text { (anhydrous) (2 mmols) } \\
+\mathrm{L}(4.2 \mathrm{mmols})\end{array}$ & $\begin{array}{l}\text { EtOH }+ \text { EtOAc, } 1: 1 \\
\text { proportion }\left(20 \mathrm{~cm}^{3}\right)\end{array}$ & $\begin{array}{l}24 \mathrm{~h} \\
\left(25^{\circ} \mathrm{C}\right)\end{array}$ & $\begin{array}{l}\text { EtOAc + petroleum } \\
\text { ether }\end{array}$ & $75-85$ \\
\hline $\mathrm{CoL}_{3} \mathrm{X}_{2}$ & $\begin{array}{l}\left.\mathrm{CoX}_{2} \text { (anhydrous) ( } 2 \text { mmols }\right) \\
+\mathrm{L}(6.3 \text { mmols })\end{array}$ & EtOH $\left(20 \mathrm{~cm}^{3}\right)$ & $\begin{array}{l}24 \mathrm{~h} \\
\left(25^{\circ} \mathrm{C}\right)\end{array}$ & $\begin{array}{l}\text { EtOAc + petroleum } \\
\text { ether }\end{array}$ & $70-80$ \\
\hline $\mathrm{NiLCl}_{2}$ & $\begin{array}{l}\mathrm{NiCl}_{2} \text { (anhydrous) (4 mmols) } \\
+\mathrm{L} \text { (4 mmoles) }\end{array}$ & EtOH $\left(20 \mathrm{~cm}^{3}\right)$ & $\begin{array}{l}24 \mathrm{~h} \\
\left(25^{\circ} \mathrm{C}\right)\end{array}$ & $\begin{array}{l}\text { EtOAc + petroleum } \\
\text { ether }\end{array}$ & 90 \\
\hline $\mathrm{NiLBr}_{2}$ & $\begin{array}{l}\mathrm{NiBr}_{2} \text { (anhydrous) (3 mmoles) } \\
+\mathrm{L}(3 \text { mmoles })\end{array}$ & EtOH $\left(20 \mathrm{~cm}^{3}\right)$ & $\begin{array}{l}24 \mathrm{~h} \\
\left(25^{\circ} \mathrm{C}\right)\end{array}$ & $\begin{array}{l}\text { EtOH + petroleum } \\
\text { ether }\end{array}$ & 90 \\
\hline $\mathrm{NiL}_{2} \mathrm{Cl}_{2}$ & $\begin{array}{l}\mathrm{NiCl}_{2} \cdot 6 \mathrm{H}_{2} \mathrm{O}(2 \mathrm{mmoles}) \\
+\mathrm{L}(4.3 \text { mmoles })\end{array}$ & EtOH $\left(20 \mathrm{~cm}^{3}\right)$ & $\begin{array}{l}12 \mathrm{~h} \\
\left(25^{\circ} \mathrm{C}\right)\end{array}$ & $\begin{array}{l}\text { EtOH }+ \text { petroleum } \\
\text { ether }\end{array}$ & 80 \\
\hline $\mathrm{NiL}_{2} \mathrm{Br}_{2}$ & $\begin{array}{l}\mathrm{NiBr}_{2} \text { (anhydrous) (3 mmoles) } \\
+\mathrm{L}(6.2 \text { mmoles })\end{array}$ & $\mathrm{MeOH}\left(20 \mathrm{~cm}^{3}\right)$ & $\begin{array}{l}12 \mathrm{~h} \\
\text { (reflux) }\end{array}$ & $\begin{array}{l}\mathrm{MeOH}+\text { petroleum } \\
\text { ether }\end{array}$ & 85 \\
\hline $\mathrm{NiL}_{3} \mathrm{Cl}_{2}$ & $\begin{array}{l}\mathrm{NiCl}_{2} \cdot 6 \mathrm{H}_{2} \mathrm{O}(2 \text { mmoles }) \\
\mathrm{L}(6.5 \text { mmoles })\end{array}$ & $\mathrm{EtOH}+\operatorname{EtOAc}\left(20 \mathrm{~cm}^{3}\right)$ & $\begin{array}{l}24 \mathrm{~h} \\
\left(25^{\circ} \mathrm{C}\right)\end{array}$ & ${ }^{\mathrm{b}} \mathrm{EtOAc}$ & 90 \\
\hline $\mathrm{NiL}_{3} \mathrm{Br}_{2}$ & $\begin{array}{l}\left.\mathrm{NiBr}_{2} \text { ( } 2 \text { mmoles }\right) \\
\mathrm{L}(6.4 \text { mmoles })\end{array}$ & $\mathrm{EtOH}+\mathrm{EtOAc}\left(20 \mathrm{~cm}^{3}\right)$ & $\begin{array}{l}24 \mathrm{~h} \\
\left(25^{\circ} \mathrm{C}\right)\end{array}$ & $\begin{array}{l}\text { EtOAc }+ \text { petroleum } \\
\text { ether }\end{array}$ & 90 \\
\hline $\mathrm{Ni}_{2} \mathrm{~L}_{3} \mathrm{Cl}_{2}$ & $\begin{array}{l}\left.\mathrm{NiCl}_{2} \text { ( } 2 \text { mmoles }\right) \\
\mathrm{L}(4 \text { mmoles })\end{array}$ & EtOH $\left(20 \mathrm{~cm}^{3}\right)$ & $\begin{array}{l}24 \mathrm{~h} \\
\text { (reflux) }\end{array}$ & $\begin{array}{l}\text { EtOH + petroleum } \\
\text { ether }\end{array}$ & 70 \\
\hline
\end{tabular}

